\title{
$\mathrm{Al}_{2} \mathrm{O}_{3} / \mathrm{PDMS}$ 复合材料热传导的分子动力学模拟
}

\author{
杜英喆张恒苑世领* \\ (山东大学化学与化工学院 济南 250100)
}

\begin{abstract}
摘要 采用分子动力学模拟方法研究了 $\mathrm{Al}_{2} \mathrm{O}_{3} /$ 聚甲基二硅氧烷(PDMS)复合材料在 $300 \mathrm{~K}$ 时的传热行为, 通过分析热传 导、温度梯度以及导热增强等理论数据, 讨论了不同半径以及不同浓度的 $\mathrm{Al}_{2} \mathrm{O}_{3}$ 填料粒子对 PDMS 传热的影响. 结果 表明随着体积分数的增大, $\mathrm{Al}_{2} \mathrm{O}_{3} / \mathrm{PDMS}$ 复合材料的热传导先减小后增加. 并且当 $\mathrm{Al}_{2} \mathrm{O}_{3}$ 填充粒子的半径为 $5 \mathrm{~nm}$ 时, $\mathrm{Al}_{2} \mathrm{O}_{3} / \mathrm{PDMS}$ 复合材料的热传导达到最大值. 通过考察传热过程中的 $\mathrm{Al}_{2} \mathrm{O}_{3}$ 填料和 $\mathrm{Al}_{2} \mathrm{O}_{3} / \mathrm{PDMS}$ 复合材料的温度和结构 变化, 添加有小半径 $\mathrm{Al}_{2} \mathrm{O}_{3}$ 填料的 $\mathrm{Al}_{2} \mathrm{O}_{3} / \mathrm{PDMS}$ 复合材料在传热的过程中主要以声子的形式进行传热, 此时决定热导率 的主要因素有热容 $c$ 、声子的平均速度 $v$ 和声子的平均自由程 $l$. 同时, 当体积分数为 $15 \%$ 时, 传导链形成, 热量可以顺 利通过 $\mathrm{Al}_{2} \mathrm{O}_{3}$ 填料形成的传导链传播, 有助于热传导的增加.
\end{abstract}

关键词 分子动力学模拟; 热传导; 聚二甲基硅氧烷; $\mathrm{Al}_{2} \mathrm{O}_{3}$ 填料; 复合材料; 热界面材料

\section{Molecular Dynamics Simulation of Thermal Conductivity of $\mathrm{Al}_{2} \mathrm{O}_{3} /$ PDMS Composites}

\author{
Yingzhe Du Heng Zhang Shiling Yuan* \\ (School of Chemistry and Chemical Engineering, Shandong University, Jinan 250100)
}

\begin{abstract}
Molecular dynamics simulation was carried out to study the heat transfer behavior of the $\mathrm{Al}_{2} \mathrm{O}_{3} /$ polydimethylsiloxane (PDMS) composites, through analyzing the thermal conductivity, temperature gradient and the thermal conductivity enhancement, we discussed the effect of the radius and volume content on the thermal conductivity of $\mathrm{Al}_{2} \mathrm{O}_{3} / \mathrm{PDMS}$ composites. The results show that the thermal conductivity of the $\mathrm{Al}_{2} \mathrm{O}_{3} / \mathrm{PDMS}$ composites decreases first and then increases. Besides, when the particle radius is $5 \mathrm{~nm}$, the thermal conductivity of $\mathrm{Al}_{2} \mathrm{O}_{3} / \mathrm{PDMS}$ composites attains the largest, which is higher than that with same volume content. In addition, by investigating the temperature and structure changes during the heat transfer behavior, we also found that the heat transfer of $\mathrm{Al}_{2} \mathrm{O}_{3} / \mathrm{PDMS}$ composites with smaller $\mathrm{Al}_{2} \mathrm{O}_{3}$ fillers is mainly conducted in the form of phonons, the main influence factors that determine the thermal conductivity are heat capacity $c$, average velocity $v$ and average free path $l$. Meanwhile, when the volume content is $15 \%$, the conduction chains occur, the thermal can transfer successfully through the conduction chains formed by the $\mathrm{Al}_{2} \mathrm{O}_{3}$ composites, which is beneficial to the increases of the thermal conductivity. We also observed the change of the structure and surface meshes of the $\mathrm{Al}_{2} \mathrm{O}_{3} / \mathrm{PDMS}$ composites with the increasing of the volume content. The $\mathrm{Al}_{2} \mathrm{O}_{3}$ fillers increase, PDMS chains distribute separately, and the pore structure increases, promoting the rigidity of the molecules. Moreover, when the volume content is $15 \%$, the PDMS chains are wrapped around the $\mathrm{Al}_{2} \mathrm{O}_{3}$ fillers, forming a PDMS- $\mathrm{Al}_{2} \mathrm{O}_{3}$ composite conductor, which enhances the synergistic effect of heat transfer, further explaining the conclusion that the $\mathrm{Al}_{2} \mathrm{O}_{3} / \mathrm{PDMS}$ composites with higher volume content has higher heat conduction. The whole system has similar change in the structure: i) with the increasing of the filler radius, the strip structure decreases, and the sphere structure increases. ii) the viewing of the partial enlarged drawing leads to the idea that the contribution of $\mathrm{Al}_{2} \mathrm{O}_{3}$ fillers to the conduction path increases with the increasing of the radius. iii) when the fillers are small, the distribution is more dispersed.
\end{abstract}

Keywords molecular dynamics simulation; thermal conductivity; polydimethylsiloxane; $\mathrm{Al}_{2} \mathrm{O}_{3}$ fillers; composites; thermal interface materials

\section{1 引言}

近年来, 由于电子产品使用频率的增加, 电子元器 件的损耗程度也逐渐增加. 根据相关文献描述, 当电子 器械的整体温度每升高 $10{ }^{\circ} \mathrm{C}$, 其损耗程度加倍; 并且 温升 $50{ }^{\circ} \mathrm{C}$ 时电子器械的寿命只有温升 $25{ }^{\circ} \mathrm{C}$ 时的
$1 / 5^{[1-2]}$. 除此之外，高温对电子产品的影响也会导致工 作寿命短、性能差. 因此, 为了提高电子产品的寿命, 保 证电子产品的可靠性和稳定性, 需要将电子元器件工作 时产生的热量进行有效地散热、冷却, 从而保证温度在 正常工作范围内.

*E-mail: shilingyuan@sdu.edu.cn

Received March 19, 2021; published May 12, 2021.

Supporting information for this article is available free of charge via the Internet at http://sioc-journal.cn.

Project supported by the Shandong University multidisciplinary research and innovation team of young scholars (2020QNQT018).

山东大学青年交叉科学群体项目(2020QNQT018). 
热界面材料(TIMs)在散热方面起着重要的作用. 具 体来说, 热界面材料最大限度地降低由于两个匹配组件 表面不匹配造成的空间间隙 ${ }^{[3]}$. 为了消除这些间隙和接 触热阻, 热界面材料通过具有高导热系数的材料来填充 几何缺陷 ${ }^{[4-6]}$. 在几种常见的热界面材料中, 聚二甲基硅 氧烷(PDMS)由于具有较高的热传导和较好的弹性经常 被用于热界面材料中. 然而, 纯 PDMS 的导热系数不高 $\left(10^{-1} \sim 10^{0} \mathrm{~W} /(\mathrm{m} \cdot \mathrm{K})\right)$. 因此, 许多粒子和填料填充到 PDMS 中来提高其热传导 ${ }^{[7-10]}$. 比如金属粉末、石墨、

$\mathrm{Al}_{2} \mathrm{O}_{3} 、 \mathrm{SiC}$ 和 $\mathrm{ZnO}$ 等.

冯奕钰等 ${ }^{[11]}$ 研究了一种自修复聚合物/碳纳米自组 装材料的热传导. Yang 等 ${ }^{[12]}$ 应用 APP 改性氮化铝(AIN) 粉作为填料, 提高了 AIN/PDMS 复合材料的导热性能, 并得到半固化 APP 改性试样的导热系数高于完全固化 试样的结论. Lee 等 ${ }^{[13]}$ 研究了 $\mathrm{Al}_{2} \mathrm{O}_{3}$ 和 $\mathrm{ZnO}$ 填料对硅橡 胶硫化特性、热性能和力学性能的影响. 黄政强等 ${ }^{[14]}$ 利 用三维混合硣制备了高导热性能的 PDMS 基复合材料, 并发现填充有体积分数 $20 \%$ boron nitride@15silver nanowires (BN@15AgNWs)杂化填料的 PDMS 基复合材 料的导热系数为 $0.914 \mathrm{~W} /(\mathrm{m} \cdot \mathrm{K})$, 是纯 PDMS 的 5.05 倍, 同时也比填充体积分数 $20 \% \mathrm{BN}$ 的 PDMS 基复合材料的 热传导高 $23 \%$. 然而, 一定填料的加入会对 PDMS 产生 不利影响. 按照制备流程, 一般将导热高分子大致分为 本征型和填充型. 当体系内不存在导热粒子时, 即为本 征型. 本征型体系的热导率, 即为本征热导率, 本征热 导率是最重要的热物性参数. 其中, Yang 等课题组为了 测量 MWNT 的本征热导率, 对同一根样品多次测量不 同长度内的热导率, 并通过比较, 排除了接触热阻等因 素的影响 ${ }^{[15-16]}$. 除此之外, 向聚合物中加入这类填料会 使得聚合物导电性增强, 因此不适用于存在电绝缘体的 电场, 同时, 这种强导电性会导致电子元件的短路 ${ }^{[17-21]}$. 一些填料也会降低聚合物的其他性质, 比如强度和弹 性, 并且让生产加工过程变得更加困难. 因此, 为了提 高聚合物的导热性能, 同时尽量减少对聚合物其它性能 的影响, 要选择合适的填料.

科学家们对填充了颗粒的 PDMS 有不同的研究. 通 常来说, 有两种典型的体积分数不同的体系, 一种是分 散体系, 该体系中的粒子几乎不接触, 体积分数相对较 高 ${ }^{[22]}$; 另一种是附着体系, 该体系中的粒子有助于聚合 物导电链的形成, 从而提高了体系的热传导 ${ }^{[23-24]}$. 然而, 附着体系会影响抗拉强度和断裂伸长率. 尤其是, 当体 积分数超过 $24.1 \%$ 的时候, 断裂伸长率大大降低, 硬度 增加, 这些都对弹性体有不利的影响, 同时也会导致其 使用价值的丧失. 另一个低含量更适用于本工作的原因 是当负载小于 $10 \%$ 时, 理论数据和实验数据之间可以获 得较好的一致性 ${ }^{[25]}$. 因此, 为了减小一些性质(使用价 值、断裂伸长率和硬度等 $)^{[26]}$ 的损失, 保持理论和实验数 据的一致性, 在此选用分散体系进行模拟研究.
本工作使用分子动力学模拟研究了 $\mathrm{Al}_{2} \mathrm{O}_{3} / \mathrm{PDMS}$ 的 热传导以及填料体积分数和颗粒大小对传热性能的影 响. 使用 LAMMPS 软件 ${ }^{[27-28]}$ 对热力学性质和输出的轨 迹信息进行了分析和统计. 本研究不仅可以为提高 PDMS 的热传导提供理论依据, 还可以解决 $\mathrm{Al}_{2} \mathrm{O}_{3}$ 填充 PDMS 存在加工难度的问题.

\section{2 计算方法}

\section{1 分子动力学模拟}

使用 LAMMPS 软件和 Material Studio (MS)软件 ${ }^{[29]}$ 对模拟体系进行了计算。首先, 利用 MS 软件中 Amorphous Cell 和 Building Tools 模块进行无定形 PDMS 和 $\mathrm{Al}_{2} \mathrm{O}_{3}$ 填料的构建. 硅氧链聚合物为 PDMS 的主要构 成成分, 其单体由 2 个硅原子、 2 个氧原子和 4 个甲基 构成，总质量为 148，聚合物链的末端设置为甲基. 采 用 MS 软件中 Forcite 模块进行初始单体的退火操作和无 定形 PDMS 几何优化操作 ${ }^{[30-31]}$. 其中, 模拟选择 Condensed-phase Optimized Molecular Potentials for Atomistic Simulation Studies (COMPASS)力场, 模拟温度 为 $300 \mathrm{~K}$, 用 Smart Minimizer 方法进行能量最小化, 收 玫级别设置为超精细. 时间步长为 $1 \mathrm{fs}$, 模拟退火总共 循环 5 次, 初始温度为 $300 \mathrm{~K}$, mid-cycle temperature 设置 为 $500 \mathrm{~K}$, 总共模拟 5000 步. 为了研究填料对 PDMS 热 传导的影响, 向 PDMS 中添加了不同半径的 $\mathrm{Al}_{2} \mathrm{O}_{3}$ 填料. $\mathrm{Al}_{2} \mathrm{O}_{3}$ 填充颗粒和初始结构如图 1 所示. 所有模拟体系 在 $z$ 方向的长度为 $9 \mathrm{~nm}$. a)

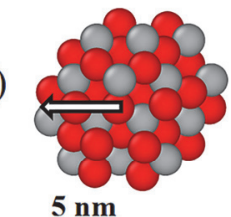

$5 \mathrm{~nm}$

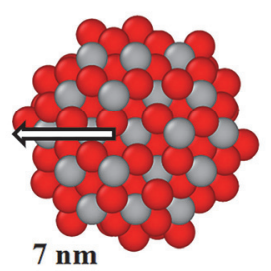

b)
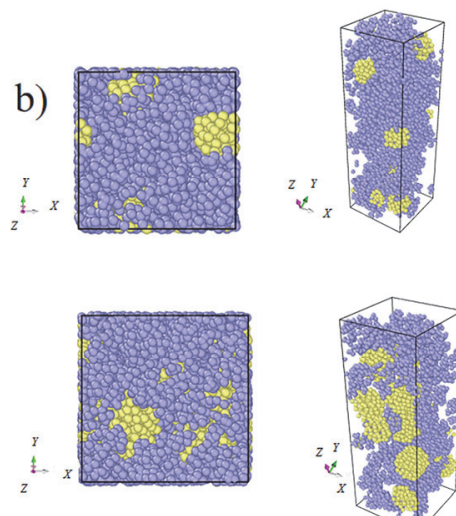

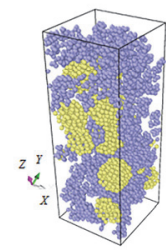

图 $1 \mathrm{Al}_{2} \mathrm{O}_{3}$ 填充颗粒和 $\mathrm{Al}_{2} \mathrm{O}_{3} / \mathrm{PDMS}$ 复合材料初始构型图. (a) $\mathrm{Al}_{2} \mathrm{O}_{3}$ 填充粒子, (b) 初始构型. 红色代表氧原子, 灰色代表铝原子, 紫色代 表 PDMS 链骨架, 黄色代表三氧化二铝填充粒子

Figure 1 Molecular structures of $\mathrm{Al}_{2} \mathrm{O}_{3}$ particles and $\mathrm{Al}_{2} \mathrm{O}_{3} / \mathrm{PDMS}$ composites. (a) $\mathrm{Al}_{2} \mathrm{O}_{3}$ fillers, (b) initial structure. Red represents oxygen atoms, gray represents aluminum atoms, purple represents PDMS skeleton, yellow represents $\mathrm{Al}_{2} \mathrm{O}_{3}$ particles

将初始结构文件转换成 LAMMPS 软件可读取的数 据文件后，使用正则系综(NVT)操作对结构进行了两次 的退火操作，使原子可以跨过在低温下不能跨过的势 垒: 从 $300 \mathrm{~K}$ 逐渐加热到 $1000 \mathrm{~K}$, 然后逐步降温, 从 
$1000 \mathrm{~K}$ 逐步冷却到 $300 \mathrm{~K}$. 在退火操作结束后, 又运行 了一次 NVT 恒温操作使体系温度控制在 $300 \mathrm{~K}$. 阻尼系 数为 $5.0 \mathrm{fs}$, 时间步长设置为 $1.0 \mathrm{fs}$, 退火操作总共运行

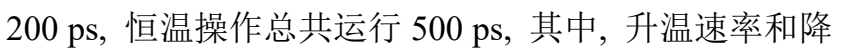
温速率皆为 $7 \mathrm{~K} / \mathrm{ps}$, 间隔 $1 \mathrm{ps}$ 记录一次轨迹. 两个 Langevin 控温器被施加在模拟箱的两端, 即冷区和热 区. 通过控制两个区域的温度, 从冷区离开的能量进入 热区, 从而形成热流密度. 在模拟中, 热区的温度为 $400 \mathrm{~K}$, 冷区的温度为 $200 \mathrm{~K}$. 在热传导的计算中, 一般 是运用声子 Boltzmann 传输方程计算和分子动力学模拟 计算, 其中分子动力学方法比声子 Boltzmann 传输方程 更加简单，在计算微尺度效应时非常有前景. 分子动力 学方法的关键是选取原子之间势函数, 势函数表征电子 云重叠的量子力学作用, 集成的势函数组成了力场, 其 中 COMPASS 力场被广泛用于热传导的计算. 此外, 由 于 COMPASS 力场是发展起来的适合凝聚态应用的一 个全新力场, 具有更多的优点和先进性, 并且通过拟合 和校正第一原理性计算数据和实验测量结果, 可以得到 具有高准确性的 COMPASS 力场. 因此本文的分子构型 优化以及热传导计算均使用 COMPASS 力场. COMPASS 力场的势函数如下:

$$
E=\varepsilon\left[2\left(\frac{\sigma}{r}\right)^{9}-3\left(\frac{\sigma}{r}\right)^{6}\right]
$$

在公式中, $E$ 为势能, $\varepsilon$ 为作用强度, $\sigma$ 与原子间平衡间距 有关, $r$ 是原子间的距离.

所有的力场参数见支持信息.

在各个方向上施加了周期性边界条件, 时间步长为 $0.5 \mathrm{fs}$, 总模拟时间为 $1 \mathrm{~ns}$. Lennard-Jones 相互作用的截 断半径为 $12.5 \mathrm{~nm}$. 使用 Ovito 软件对模拟结果进行可视 化分析. $\mathrm{Al}_{2} \mathrm{O}_{3}$ 填料的半径和体积分数列于表 1 中. PDMS 的链长为 $46.5 \mathrm{~nm}$, 所有体系的密度为 0.963 $\mathrm{g} / \mathrm{cm}^{3}[32]$.

表 $1 \mathrm{Al}_{2} \mathrm{O}_{3}$ 填料的半径和体积分数

Table 1 The size and volume content (\%) of $\mathrm{Al}_{2} \mathrm{O}_{3}$ filler

\begin{tabular}{ccccc}
\hline Particle radii $/ \mathrm{nm}$ & \multicolumn{4}{c}{$\mathrm{Al}_{2} \mathrm{O}_{3}$ loading (volume content $/ \%$ ) } \\
\hline 5 & 5 & 10 & 15 & 20 \\
7 & & & & \\
\hline
\end{tabular}

傅里叶定律用于计算热传导 [33]

$$
\begin{aligned}
& J=-\lambda \nabla T \\
& J=\frac{\Delta E}{A \Delta \mathrm{t}}
\end{aligned}
$$

在公式(1)中, $\lambda$ 表示热传导, $J$ 表示热流密度, $\nabla T$ 表示沿 着 $z$ 轴方向的温度梯度; 在公式(2)中, $\Delta E$ 表示热区和冷 区的能量差, $A$ 表示沿着热流密度方向的有效横截面 积 ${ }^{[34-35]}, \Delta t$ 表示时间间隔.

\section{3 结果与讨论}

\section{1 体积分数对 $\mathrm{Al}_{2} \mathrm{O}_{3} / \mathrm{PDMS}$ 复合材料热传导的影响}

为了证明体系的平衡, 计算了实施退火操作后的体 系能量变化和温度变化, 结果如图 $\mathrm{S} 1$ (支持信息). 体系 能量保持在 $-6 \times 10^{5} \mathrm{~kJ} / \mathrm{mol}$ 左右, 温度保持在 $300 \mathrm{~K}$, 即理想温度，表明体系达到了稳定结构. 同时，在模拟 过程两个区域的温度都达到了稳定状态，与所设定的固 定温度一致：热区温度为 $400 \mathrm{~K}$, 冷区温度为 $200 \mathrm{~K}$. 证 明操作的合理性以及传热已进入稳定阶段.

研究表明填充粒子的体积对体系热传导也有一定 的影响 ${ }^{[36]}$, 对含有不同体积分数的 $\mathrm{Al}_{2} \mathrm{O}_{3}$ 填料的 PDMS 复合材料的热传导进行了计算. 结果如图 2 所示，可以 看出当体积分数小于 $10 \mathrm{vol} \%$ 时，热传导减小，当体积 分数大于 $10 \mathrm{vol} \%$ 时, 热传导增加, 这与吴大鸣等 ${ }^{[37]}$ 的 结果相一致. 并且当 $\mathrm{Al}_{2} \mathrm{O}_{3}$ 填料体积分数为 $10 \%$ 时, 填 充了不同半径 $\mathrm{Al}_{2} \mathrm{O}_{3}$ 填料的 PDMS 复合材料的热传导达 到了最小值. 我们推测当 $\mathrm{Al}_{2} \mathrm{O}_{3}$ 体积分数小于 $10 \%$ 时, 两个相邻的 $\mathrm{Al}_{2} \mathrm{O}_{3}$ 颗粒距离相对较远, 因此存在一种弱 接触降低了填料传导链形成的可能性; 但是当填料的体 积分数大于 $10 \mathrm{vol} \%$ 时，相邻的 $\mathrm{Al}_{2} \mathrm{O}_{3}$ 颗粒之间距离较 近，这种填料之间的紧密连接有助于声子和电子传导路 径的形成, 从而提高热传导 ${ }^{[32]}$. 除此之外, 当体积分数 为 $10 \%$ 时, 含有 $5 \mathrm{~nm} \mathrm{Al}_{2} \mathrm{O}_{3}$ 填料的 PDMS 复合材料的热 传导大于含有 $7 \mathrm{~nm} \mathrm{Al}_{2} \mathrm{O}_{3}$ 填料的 PDMS 复合材料的热传 导; 当体积分数为 $20 \%$ 时, 热传导顺序也是如此.

当比较聚合物和纯 PDMS 热传导时, 导热增强 (TCE)也是另一影响因素，计算公式如下 ${ }^{[38]}$ :

$$
T C E=\frac{K_{\mathrm{c}}-K_{\mathrm{m}}}{K_{\mathrm{m}}}
$$

在公式(3)中, $K_{\mathrm{c}}$ 表示 $\mathrm{Al}_{2} \mathrm{O}_{3} / \mathrm{PDMS}$ 复合材料的热传导, $K_{\mathrm{m}}$ 表示 PDMS 基体的热传导.

图 2(b) 显示了填充了不同体积分数的 $\mathrm{Al}_{2} \mathrm{O}_{3}$ 的 PDMS 复合材料的导热增强. 可以发现当体积分数为 $10 \%, 15 \%$ 和 $20 \%$ 时，填充有 $5 \mathrm{~nm} \mathrm{Al}_{2} \mathrm{O}_{3}$ 填料的 $\mathrm{Al}_{2} \mathrm{O}_{3} / \mathrm{PDMS}$ 复合材料的导热增强总是低于填有其他半 径的复合材料的导热增强. 并且当 $\mathrm{Al}_{2} \mathrm{O}_{3}$ 填充体积分数 为 $10 \%$ 时, $\mathrm{Al}_{2} \mathrm{O}_{3} / \mathrm{PDMS}$ 复合材料的 $\mathrm{TCE}$ 值分别为 $-62.939 \mathrm{vol} \%,-82.568 \mathrm{vol} \%$. 另外, 对于填充了较低 体积分数的复合材料，填料完全被聚合物基体包覆，填 料与聚合物基体之间的边界热阻 $\left(R_{\mathrm{b}}\right)$ 是影响导热填料及 其影响性能的主要阈值, 根据文献显示, 较低的 $R_{\mathrm{b}}$ 有利 于热传导. 从图 3(b) 可以看出, 半径为 $5 \mathrm{~nm}$ 的 $\mathrm{Al}_{2} \mathrm{O}_{3}$ 填 料在提高热传导和降低 $R_{\mathrm{b}}$ 方面比其他半径的 $\mathrm{Al}_{2} \mathrm{O}_{3}$ 表现 出更好的性能, 说明填料尺寸是影响填料与 PDMS 界面 的重要因素. 
a)

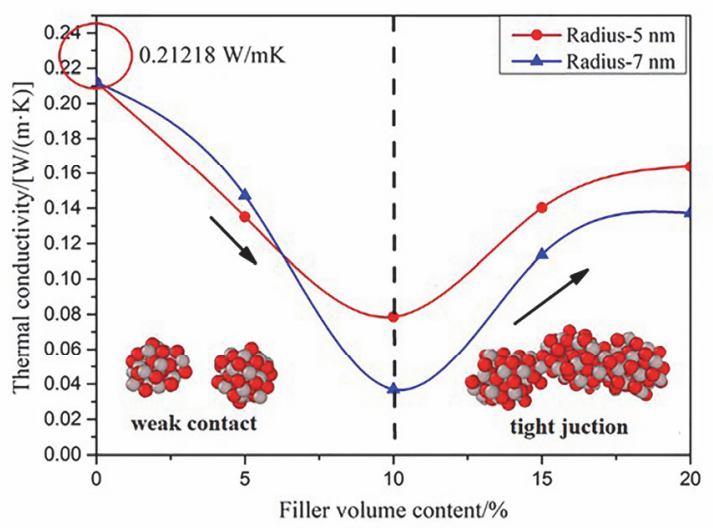

b)

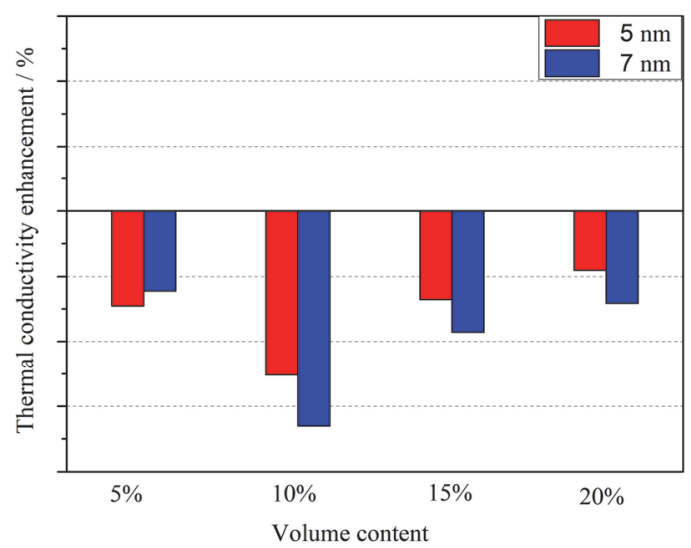

图 2 不同体积分数的复合材料传热性能的变化. (a) 导热系数. (b) TCE

Figure 2 The changes in the thermal properties with different filler volume contents. (a) Thermal conductivity. (b) TCE

\section{2 填料半径对 $\mathrm{Al}_{2} \mathrm{O}_{3} / \mathrm{PDMS}$ 热传导的影响}

以上结论证明，当体积分数大于 $10 \%$ 时, $\mathrm{Al}_{2} \mathrm{O}_{3} / \mathrm{PDMS}$ 复合材料的热传导增加, 在此基础上, 对 填料半径对复合材料热传导的影响进行了考察(图 3). 结果表明, 随着颗粒半径的增大, 热传导逐渐降低, 在 相同的体积分数下, 填充物较小的半径提高了复合材料 的热传导性能, 这与周文英等 ${ }^{[39}$ 得到的结论相一致. 除 此之外, 通过对整个计算结果进行考察, 不同体积分数 对热传导也有不同的变化趋势: 体积分数大于 $10 \%$ 时, $\mathrm{Al}_{2} \mathrm{O}_{3}$ 填料体积分数越大, 热传导越高.

为了研究这一现象, 进一步对纯 PDMS 基体和 $\mathrm{Al}_{2} \mathrm{O}_{3} / \mathrm{PDMS}$ 复合材料的温度和粒子类型分布进行了分 析. 以含有半径为 $7 \mathrm{~nm}$, 体积分数为 $10 \%$ 的 $\mathrm{Al}_{2} \mathrm{O}_{3}$ 的复 合材料为例进行讨论. 如图 4 所示, 热区位于 $1 \mathrm{~nm}$ 与 2 $\mathrm{nm}$ 之间, 温度为 $200 \mathrm{~K}$, 冷区位于 $8 \mathrm{~nm}$ 与 $9 \mathrm{~nm}$ 之间, 温 度为 $400 \mathrm{~K}$. 此外, 可以发现在 $1 \mathrm{~nm}$ 至 $3 \mathrm{~nm}$ 区域, 体系 温度突然增加, 粒子类型也突然发生变化, 说明在这个 区域有新的原子或新的分子出现. 通过查看最后一帧的 轨迹发现, $\mathrm{Al}_{2} \mathrm{O}_{3}$ 填料主要分布在这个区域, 造成了粒子 类型的改变. 经过对比纯 PDMS 与 $\mathrm{Al}_{2} \mathrm{O}_{3} / \mathrm{PDMS}$ 复合材 料的温度和粒子类型分布图推断, $\mathrm{Al}_{2} \mathrm{O}_{3}$ 填料是造成复
合材料局部温度升高的主要因素.

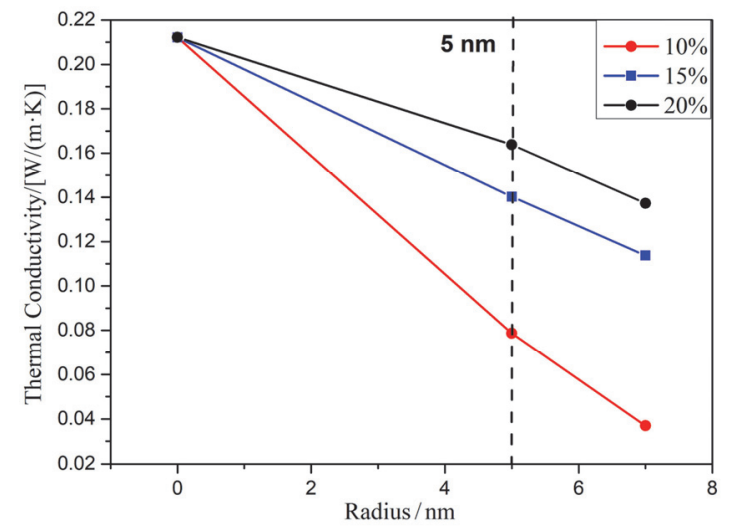

图 3 不同粒径 $\mathrm{Al}_{2} \mathrm{O}_{3} / \mathrm{PDMS}$ 复合材料的热传导

Figure 3 The thermal conductivity of $\mathrm{Al}_{2} \mathrm{O}_{3} / \mathrm{PDMS}$ composites with different particle radius

a)

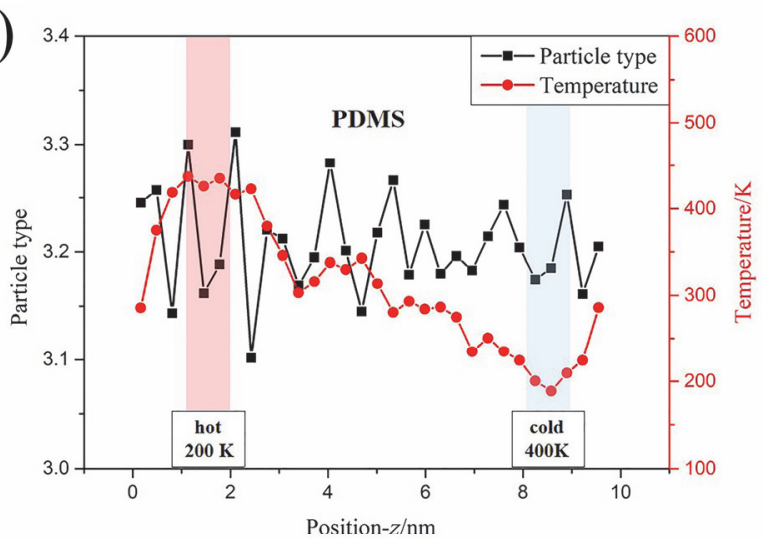

b)

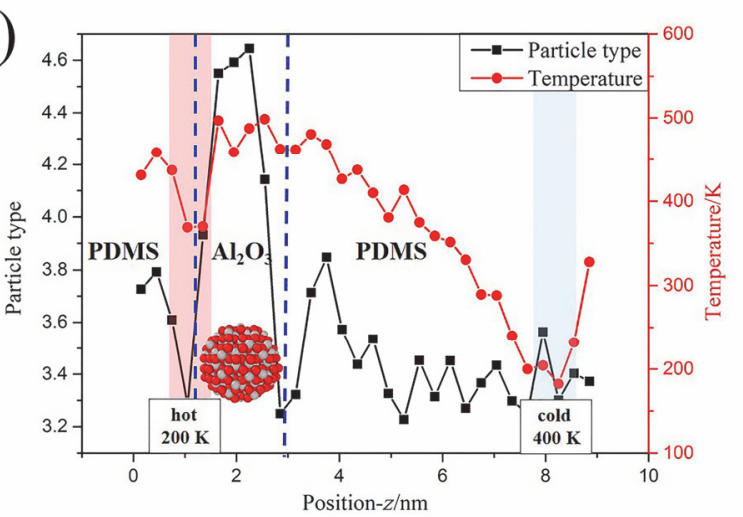

图 4 纯 PDMS 和 $\mathrm{Al}_{2} \mathrm{O}_{3} / \mathrm{PDMS}$ 复合材料的温度分布和沿 $\mathrm{z}$ 轴方向粒 子类型. (a) 纯 PDMS. (b) $\mathrm{Al}_{2} \mathrm{O}_{3} / \mathrm{PDMS}$ 复合材料

Figure 4 The temperature distribution and particle type in $z$-direction of $\mathrm{Al}_{2} \mathrm{O}_{3} / \mathrm{PDMS}$ composites and PDMS. (a) pure PDMS. (b) $\mathrm{Al}_{2} \mathrm{O}_{3} / \mathrm{PDMS}$ composites

\section{$3.3 \mathrm{Al}_{2} \mathrm{O}_{3}$ 填料}

许多研究表明选择 $\mathrm{Al}_{2} \mathrm{O}_{3}$ 和其他填料会增加 PDMS 聚合物的热传导, 但很少有人研究纯 $\mathrm{Al}_{2} \mathrm{O}_{3}$ 对 PDMS 聚 合物热传导的研究，因此我们对 $\mathrm{Al}_{2} \mathrm{O}_{3} / \mathrm{PDMS}$ 复合材料 
进行了研究.

图 5 显示了半径为 $5 \mathrm{~nm}$ 和 $7 \mathrm{~nm}$ 的 $\mathrm{Al}_{2} \mathrm{O}_{3}$ 填料在热 传导过程中的温度变化. 较小半径的颗粒在传热的过程 中温度整体较低, 而较大半径的颗粒在传热的过程中温 度整体较高, 结合温度较低时, 材料以声子热导为主的 结论, 可以推断添加有小半径 $\mathrm{Al}_{2} \mathrm{O}_{3}$ 填料的 $\mathrm{Al}_{2} \mathrm{O}_{3} / \mathrm{PDMS}$ 复合材料在传热的过程中主要以声子的形 式进行传热，导热率可以通过下式计算:

$$
\kappa=\frac{1}{3} c v l
$$

此时主要决定因素有热容 $c$ 、声子的平均速度 $v$ 和 声子的平均自由程 $l$. 一般来说, 填料的形状不同, 对热 导率的提升效果也不同 ${ }^{[40]}$. 由图可见, $\mathrm{Al}_{2} \mathrm{O}_{3}$ 填料尺寸越 大, 形状越不规则, 对传导链和导热网络的形成具有一 定的影响, 进一步说明 $\mathrm{Al}_{2} \mathrm{O}_{3}$ 填料尺寸、形状对复合材 料热传导的重要性. a)
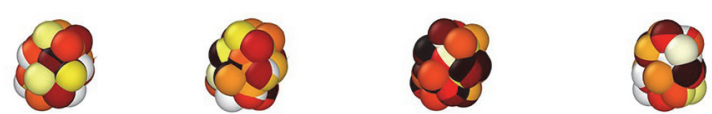

b)
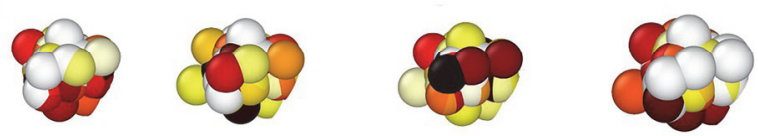

cold

hot
以发现 PDMS 链缠绕在 $\mathrm{Al}_{2} \mathrm{O}_{3}$ 传导链的周围, 形成了 $\mathrm{PDMS}-\mathrm{Al}_{2} \mathrm{O}_{3}$ 复合导体，增强了传热的协同作用，有利 于热量的传播, 也进一步解释了高体积分数的 $\mathrm{Al}_{2} \mathrm{O}_{3}$ 热 传导较高的结论.

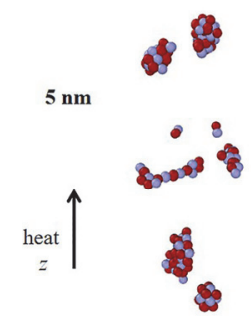

$5 \%$

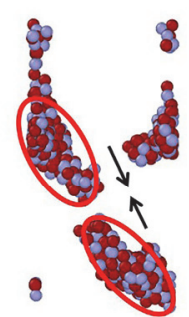

$10 \%$

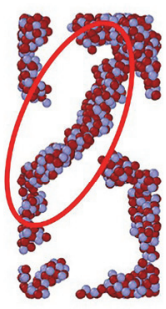

$15 \%$
图 6 半径为 $5 \mathrm{~nm}$, 不同体积分数下 $\mathrm{Al}_{2} \mathrm{O}_{3}$ 填料传导链的形成

Figure 6 The formation of the conduction chains formed by $\mathrm{Al}_{2} \mathrm{O}_{3}$ fillers of radius $5 \mathrm{~nm}$ with different volume contents
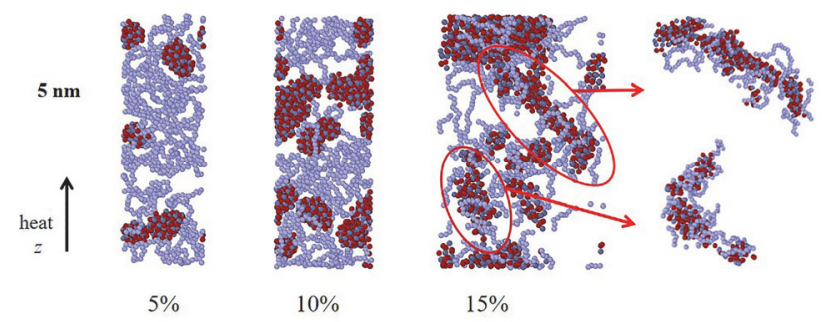

图 7 随体积分数增加的 $\mathrm{Al}_{2} \mathrm{O}_{3} / \mathrm{PDMS}$ 复合材料的结构变化图 Figure 7 The structure change of the $\mathrm{Al}_{2} \mathrm{O}_{3} / \mathrm{PDMS}$ composites with the increasing of the volume content

为了调查在 PDMS 链中加入不同半径 $\mathrm{Al}_{2} \mathrm{O}_{3}$ 填料后

图 $5 \mathrm{Al}_{2} \mathrm{O}_{3}$ 填料在热传导过程中的温度变化. (a) $5 \mathrm{~nm}$, (b) $7 \mathrm{~nm}$

Figure 5 The temperature changes of the $\mathrm{Al}_{2} \mathrm{O}_{3}$ fillers during the thermal conduction. (a) $5 \mathrm{~nm}$, (b) $7 \mathrm{~nm}$

热传导链和传导网络的形成有助于传热, 为探究 $\mathrm{Al}_{2} \mathrm{O}_{3}$ 填料对传导链形成的影响, 对半径为 $5 \mathrm{~nm}$ 的 $\mathrm{Al}_{2} \mathrm{O}_{3}$ 填料进行了探究. 由图 6 可以发现, 随着体积分数 的增加, 颗粒逐渐相互靠近形成传导链. 当体积分数为 $5 \%$ 时, $\mathrm{Al}_{2} \mathrm{O}_{3}$ 填料分布较为分散; 当体积分数为 $10 \%$ 时, 传导链开始形成, 使得热量可以顺利在传导链之间传 播; 当体积分数为 $15 \%$ 时, 传导链形成, 热量可以顺利 通过 $\mathrm{Al}_{2} \mathrm{O}_{3}$ 填料形成的传导链传播, 有助于热传导的增 加, 这也可以解释之前的结论, 即 $\mathrm{Al}_{2} \mathrm{O}_{3}$ 填料体积分数 越大, 传热越好, 热导率越高.

\section{$3.4 \mathrm{Al}_{2} \mathrm{O}_{3} / \mathrm{PDMS}$ 复合填料体系}

为了探究 $\mathrm{Al}_{2} \mathrm{O}_{3} / \mathrm{PDMS}$ 复合填料体系在整个传热过 程中在结构上的变化, 以及探讨验证其之间是否有复合 作用, 半径为 $5 \mathrm{~nm}$ 时, $\mathrm{Al}_{2} \mathrm{O}_{3} / \mathrm{PDMS}$ 复合填料随体积分 数升高时结构上变化情况, 由图 7 可以看出, 随着体积 分数的增加, $\mathrm{Al}_{2} \mathrm{O}_{3}$ 填料逐渐增多, PDMS 分子链逐渐扩 散, 孔隙状结构也逐渐增加, 增加了体系的刚性. 与此 同时, 当体积分数为 $15 \%$ 时, 通过放大局部细节图, 可
复合材料的结构差异，对在相同体积分数 $(10 \%)$ 下的整 个体系的表面网格进行了讨论研究. 在此过程中, 为了 更好地显示 $\mathrm{Al}_{2} \mathrm{O}_{3}$ 填料, 探针的半径设置为 $2 \mathrm{~nm}$, 平滑 等级设置为 8 , 粒子的半径设置为 $1.5 \mathrm{~nm}$. 从图 8 可以得 到以下结论: (1)随着半径的增加, 条状结构逐渐减少, 孔状结构减少, 球状结构增加. (2)局部放大图的观察结 果表明 $\mathrm{Al}_{2} \mathrm{O}_{3}$ 填料对导电链的形成随半径的增大而增 大. (3)局部放大图也能反映 $\mathrm{Al}_{2} \mathrm{O}_{3}$ 填料的分布: 即填料 半径越小, 分布越分散. 除此之外, 本工作对 PDMS 聚 合物链的动力学过程进行了分析, 结果如图 9 所示. 在 运动的过程中, 未缠绕在 $\mathrm{Al}_{2} \mathrm{O}_{3}$ 上的 PDMS 聚合物链运 动较为混乱无序. 而缠绕在 $\mathrm{Al}_{2} \mathrm{O}_{3}$ 上的 PDMS 聚合物链 运动较少, 较为固定. 证明了 PDMS 链可以结合在 $\mathrm{Al}_{2} \mathrm{O}_{3}$ 填料上, 增强了导热链形成的稳定性, 促进了传 热. 并且 PDMS 链在传热的过程中可以形成空间网络结 构, 也能有效缓解热应力, 对断裂韧性也有积极的影响.

\section{5 边界散射对热导率的影响}

当声子本征驰豫时间远大于声子-边界散射的弛豫 时间时, 热传导以声子-边界散射为主, 称之为弹道声 子散射; 当声子本征驰豫时间较短时, 热传导以声子本 
a)

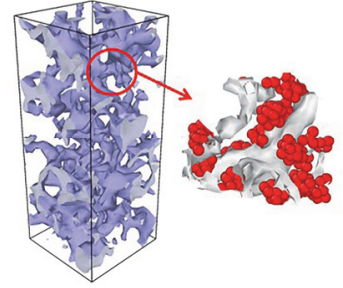

b)

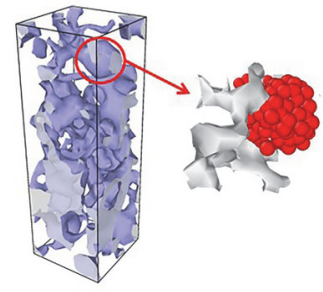

图 $8 \mathrm{Al}_{2} \mathrm{O}_{3} / \mathrm{PDMS}$ 复合材料的表面网格和局部放大图

Figure 8 The surface meshes of the $\mathrm{Al}_{2} \mathrm{O}_{3} / \mathrm{PDMS}$ composites and partial enlarged drawing

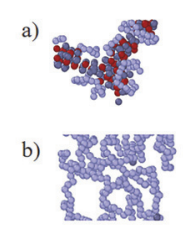

0 ps

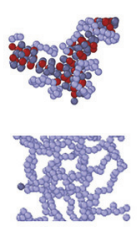

$200 \mathrm{ps}$

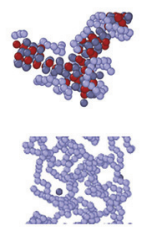

$400 \mathrm{ps}$

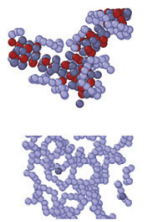

$600 \mathrm{ps}$

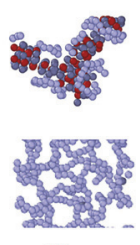

$800 \mathrm{ps}$
图 9 PDMS 链动力学过程分析. (a) 缠绕着 $\mathrm{Al}_{2} \mathrm{O}_{3}$ 的聚合物链, (b) 单 独聚合物链. 浅紫色代表 PDMS 链骨架, 红色代表氧原子, 深紫色代 表铝原子

Figure 9 The dynamics process analysis of PDMS chains. (a) PDMS chains wrapped around $\mathrm{Al}_{2} \mathrm{O}_{3}$ fillers, (b) individual PDMS chains. Light purple represents PDMS skeleton, red represents oxygen atoms, deep purple represents aluminum atoms

征散射为主, 为扩散声子散射 ${ }^{[41]}$. 随着链长的增加, 传 热方式由弹道声子传热向扩散声子传热转变. 本研究的 分子链长为 $46.5 \mathrm{~nm}$, 主要以弹道声子传热为主, 此时 分子链中的声子主要受到边界处冷、热区域散射的干扰, 传递热量较少, 热导率较低. 同时, 热传导也会受到有 限尺寸效应的影响, 当模拟的尺寸 $(L z)$ 小于声子平均自 由路径(MFP)时, 导热系数受到模拟尺寸的限制[42]. 存 在的关系如下:

$$
\frac{1}{\kappa}=\frac{2}{\kappa_{\mathrm{B}} n v}\left(\frac{1}{l_{\infty}}+\frac{4}{L}\right)
$$

在公式(4)中, $K$ 为导热率, $\kappa_{\mathrm{B}}$ 为玻尔兹曼常数; $n$ 为体 系原子的数密度, 只与材料的晶格常数有关; $v$ 为声子 的群速, 通常简化为材料中的声速; $l_{\infty}$ 为无限体系的声 子平均自由程, 仅与材料自身的非简谐特性有关; $L$ 为 链长. 由此式可以看出, 导热率随着链长的增加而增加, 并且增加的趋势逐渐变缓慢.

\section{4 结论}

本文通过运用分子动力学模拟研究了 $\mathrm{Al}_{2} \mathrm{O}_{3} / \mathrm{PDMS}$ 复合填料的热传导, 以及 $\mathrm{Al}_{2} \mathrm{O}_{3}$ 填料半径和体积分数对 复合填料热传导的影响, 结果表明, 随着体积分数的增 大, $\mathrm{Al}_{2} \mathrm{O}_{3} / \mathrm{PDMS}$ 复合材料的热传导先减小后增加. 与此 同时, 当 $\mathrm{Al}_{2} \mathrm{O}_{3}$ 填充粒子的半径为 $5 \mathrm{~nm}$ 时, $\mathrm{Al}_{2} \mathrm{O}_{3} / \mathrm{PDMS}$ 复合材料的热传导达到最大值. 通过考察传热过程中的
$\mathrm{Al}_{2} \mathrm{O}_{3}$ 填料和 $\mathrm{Al}_{2} \mathrm{O}_{3} / \mathrm{PDMS}$ 复合材料的温度和结构变化, 添加有小半径 $\mathrm{Al}_{2} \mathrm{O}_{3}$ 填料的 $\mathrm{Al}_{2} \mathrm{O}_{3} / \mathrm{PDMS}$ 复合材料在传 热的过程中主要以声子的形式进行传热, 此时决定热导 率的主要因素有热容 $c$ 、声子的平均速度 $v$ 和声子的平 均自由程 $l$. 同时, 当体积分数为 $15 \%$ 时, 传导链形成, 热量可以顺利通过 $\mathrm{Al}_{2} \mathrm{O}_{3}$ 填料形成的传导链传播, 有助 于热传导的增加, 这也可以解释之前的结论, 即 $\mathrm{Al}_{2} \mathrm{O}_{3}$ 填料体积分数越大, 传热越好, 热导率越高. 最后, 缠 绕在 $\mathrm{Al}_{2} \mathrm{O}_{3}$ 上的 $\mathrm{PDMS}$ 聚合物链较为固定, 增强了导热 链形成的稳定性，促进了体系的传热.

\section{References}

[1] Fu, G. C.; Gao, Z. X.; Fang, Z. Q.; Zou, H. Electro-mechanical Engineering 2004, 103, 13 (in Chinese). (付桂翠, 高泽溪, 方志强, 邹航，电子机械工程, 2004, 103, 13.)

[2] Gohy, J. F.; Vanhoorne, A.; Jérôme, R. Macromolecules 1996, 29, 3376.

[3] Park, W.; Guo, Y.; Li, X. J. Phys. Chem. C 2015, 119, 26753.

[4] Ralphs, M. I.; Kemme, N.; Vartak, P. B.; Joseph, E.; Tipnis, S.; Turnage, S.; Solanki, K.N.; Wang, R. Y.; Rykaczewski, K. ACS Appl. Mater. Interfaces 2018, 10, 2083.

[5] Zhang, R.; Cai, J.; Wang, Q.; Li, J.; Hu, Y.; Du, H.; Li, L. J. Electron. Packag. 2014, 136, 011012.

[6] Chung, D. D. Appl. Therm. Eng. 2001, 21, 1593.

[7] Sim, L. C.; Ramanan, S. R.; Ismail, H.; Seetharamu, K. N.; Goh, T. J. Thermochim. Acta 2005, 430, 155.

[8] Goel, P.; Choudhury, M. D.; Bin Aqeel, A.; Li, X. Y.; Shao, L. H.; Duan, H. L. Langmuir 2019, 35, 6916.

[9] Yao, Y. M.; Ye, Z. Q.; Huang, F. Y.; Zeng, X. L. ACS Appl. Mater. Interfaces 2020, 12, 2892.

[10] Pan, C. F.; Ohm, Y.; Wang, J. X.; Ford, M. J.; Kumar, K.; Kumar, S.; Majidi, C. ACS Appl. Mater. Interfaces 2019, 11, 42561.

[11] Yu, H. T.; Feng, Y. Y.; Gao, L.; Chen, C. Macromolecules 2020, 53, 7161.

[12] Yang, C. R.; Chen, C. D.; Cheng, C.; Shi, W. H.; Chen, P. H.; Teng, T. P. Int. J. Therm. Sci. 2020, 155, 106431.

[13] Sim, L. C.; Lee, C. K.; Ramanan, S. R.; Ismall, H.; Seetharamu, K. N. Polym.-Plast. Technol. Eng. 2006, 45, 301.

[14] Huang, Z. Q.; Wu, W.; Drummer, D.; Liu, C.; Wang, Y.; Wang, Z. Y. Polymers 2021, 13, 248.

[15] Yang, J. K.; Yang, Y. Small 2011, 7, 2334.

[16] Zhou, W. Y.; Zhang, Y. T. China Synthetic Resin and Plastics 2010 27, 69 (in Chinese). (周文英，张亚婷，合成树脂及材料, 2010, 27, 69.)

[17] Socher, R.; Krause, B.; Hermasch, S.; Wursche, R.; Potschke, P. Compos. Sci. Technol. 2011, 71, 1053.

[18] Cai, S. Y.; Chang, C. H.; Huang, Y. F.; Lin, W. J. ACS Appl. Mater. Interfaces 2018, 10, 17393.

[19] Pak, S. Y.; Kim, H. M.; Kim, S. Y.; Youn, J. R. Carbon 2012, 50 , 4830 .

[20] Xie, B. H.; Huang, X.; Zhang, G. J. Compos. Sci. Technol. 2013, 85, 98.

[21] Yang, S. Q.; Li, W. Z.; Bai, S. B. ACS Appl. Mater. Interfaces 2019 , 11,3388 .

[22] Lin, F.; Bhatia, G. S.; Ford, J. D. J. Appl. Polym. Sci. 1993, 49, 1869.

[23] Nielsen, L. E. J. Appl. Polym. Sci. 1973, 17, 3819.

[24] Agari, Y.; Uno, T. J. Appl. Polym. Sci. 1986, 32, 5705.

[25] Plimpton, S. J. Comput. Phys. 1995, 117, 1.

[26] Mu, Q. H.; Feng, S. Y.; Diao, G. Z. Polym. Composite. 2010, 28, 125.

[27] Aktulga, H. M.; Fogarty, J. C.; Pandit, A. Y. Parallel Comput. 2012, $38,245$.

[28] Yuan, S. D.; Wang, X. Y.; Zhang, H. J. Phys. Chem. C 2020, 124, 1932.

[29] Moradi, H.; Azizpour, H.; Bahmanyar, H. Heliyon 2020, 6, e05383.

[30] Wang, Z. T.; Li, H.; Yan, S. C.; Fang, Q. R. Acta Chim. Sinica 2020, 78,63 (in Chinese). (王志涛, 李辉, 颜士臣, 方千荣, 化学学报, 2020, 78, 63.) 
[31] Yu, H.; Guo, J.; Wang, J.; Long, Y. C. Acta Chim. Sinica 2012, 70, 1543 (in Chinese). (余辉, 郭娟, 汪靖, 龙英才, 化学学报, 2012, 70, 1543.)

[32] Xu, W. X.; Wu, Y. Y.; Zhu, Y.; Liang, X. G. Chin. Phys. B 2020, 29, 046601 (in Chinese). (徐文雪, 吴雁艳, 祝洣, 梁新刚, 中国物理 B, 2020, 29, 046601.)

[33] Mark, J. J. Am. Chem. Soc. 2009, 131, 16330.

[34] Castejon, H. J. J. Phys. Chem. B 2003, 107, 826.

[35] Ikeshoji, T.; Hafskjold, B. Mol. Phys. 1994, 81, 251

[36] Sundstrom, D. W.; Lee, Y. D. J. Appl. Polym. Sci. 1972, 16, 3159.

[37] Si, W. Y.; He, X. X.; Huang, Y.; Gao, X. L.; Zheng, X. T.; Zheng, X. P.; Leng, C.; Su, F. C.; Wu, D. M. RSC Adv. 2018, 8, 36007.
[38] Li, A.; Zhang, C.; Zhang, Y. F. Polymers 2017, 9, 437.

[39] Zhou, W. Y.; Qi, S. H.; Tu, C. C.; Zhao, H. Z.; Wang, C. F.; Kou, J. L. J. Appl. Polym. Sci. 2010, 104, 1312.

[40] Hao, L. Y.; Wen, B. Y.; Zhang, Y. P. China Plastics 2017, 31, 1994 (in Chinese). (郝鲁阳, 温变英, 张宜鹏, 中国塑料, 2017, 31, 1994.)

[41] Liu, J.; Yang, R. G. Phys. Rev. B 2012, 86, 104307.

[42] Schelling, P. K.; Phillpot, S. R.; Keblinski, P. Phys. Rev. B 2002, 65, 144306.

(Cheng, B.) 